\title{
Network Pharmacology-Based Strategy for Predicting Active Ingredients and Potential Targets of Gegen Qinlian Decoction for Rotavirus Enteritis
}

\author{
Peicheng Zhong $\mathbb{D}^{1}{ }^{1}$ Lijun Song, ${ }^{1,2}$ Mengyue Gao, ${ }^{1}$ Xiaotong Wang, ${ }^{1}$ Wenpan Tan, \\ Huanqian Lu, ${ }^{1}$ Qian Lan $\mathbb{D}^{1},{ }^{1}$ Zuyi Zhao, ${ }^{3}$ and Wenchang Zhao $\mathbb{D}^{1,2}$ \\ ${ }^{1}$ School of Pharmacy, Guangdong Medical University, Dongguan 523808, Guangdong, China \\ ${ }^{2}$ Guangdong Key Laboratory for Research and Development of Natural Drugs, Guangdong Medical University, \\ Zhanjiang 524023, Guangdong, China \\ ${ }^{3}$ School of Mathematics, Sun Yat-Sen University, Guangzhou 510275, Guangdong, China
}

Correspondence should be addressed to Wenchang Zhao; zhaowenchang@126.com

Received 9 April 2020; Revised 28 June 2020; Accepted 13 July 2020; Published 30 July 2020

Academic Editor: Vincenzo De Feo

Copyright (c) 2020 Peicheng Zhong et al. This is an open access article distributed under the Creative Commons Attribution License, which permits unrestricted use, distribution, and reproduction in any medium, provided the original work is properly cited.

\begin{abstract}
Ethnopharmacological Relevance. Gegen Qinlian decoction (GGQLD) is an effective formula treatment for rotavirus enteritis (RVE), which has been applied for 1900 years. It consists of 4 herbal medicines corresponding to the four roles "monarch, minister, assistant, and guide," which is the basic rule of prescription composition in traditional Chinese medicine (TCM). However, its active ingredients and therapeutic mechanism on RVE have not been fully investigated. Materials and Methods. In this study, a network pharmacology-based strategy was used to elucidate the mechanism of GGQLD for the treatment of RVE. Oral bioavailability and drug-likeness were taken as the judgment criteria to search the active ingredients of GGQLD in traditional Chinese medicine systems pharmacology database and analysis platform (TCMSP). The affinity between protein and ingredients was further determined using the similarity ensemble approach to find the corresponding targets. According to the genes related to enteritis in GeneCards database, the key targets were screened by intersections between drug and disease targets. And the therapeutic mechanism was predicted using the protein-protein interactions (PPIs), the Gene Ontology (GO), and the Kyoto Encyclopedia of Genes and Genomes (KEGG) database, which was verified by detecting calcium ion concentration with the fluorescent probe. Result. 130 active ingredients were screened from GGQLD, including (R)-canadine, moupinamide, formononetin, and other flavonoids. They act on a total of 366 targets, which is mainly distributed in the biological process of hormone binding or signaling pathways of neuroactive ligand receptor interaction, serotonergic synapse, and calcium signaling pathway. Furthermore, serotonin receptors, adrenergic receptors, cholinergic receptors, and dopamine receptors in the enteric nervous system may be the key targets of RVE treatment by GGQLD. Conclusion. This study demonstrated that the potential mechanism that GGQLD can effectively improve the symptoms of RVE may depend on the regulation of calcium ions, serotonin, and gastrointestinal hormone ion that could mutually affect the intestinal nervous system.
\end{abstract}

\section{Introduction}

Traditional Chinese medicine (TCM), which has been widely used in clinical practice in China for thousands of years, takes multimedicinal materials compatibility for treating complex diseases [1]. And TCM formulas could be considered a complex system that consists of hundreds of chemical compounds, which exert synergistic, mutual assistance therapeutic action with fewer side effects on the complex giant system of the human body [2]. Thus, it presents a serious challenge to explain the mechanism of action of TCM formulas.

Network pharmacology is a systematic analytical method based on the interaction network of diseases, genes, protein 
targets, and drugs to elucidate the mechanism of drug action [3]. With the help of computational network pharmacology, it could explain TCM formulas' acting characteristics of integrity, synergy, and dynamic in a holistic view and affords new sights into the multicomponent and multitargeted therapeutics of TCM. Therefore, network pharmacology holds a powerful and promising tool for analyzing TCM formulas.

GGQLD, a four-herb Chinese medicine formula first described 1900 years ago, is composed of Pueraria lobate, Scutellaria baicalensis, Coptis chinensis, and Glycyrrhiza uralensis. It has definite curative effect on RVE $[4,5]$, which is characterized by diarrhea, vomiting, fever, and so on. More than 200,000 infant deaths by RV are noted annually without specific drugs in clinic [6]. But the complex composition of GGQLD makes it difficult to conduct molecular mechanistic research on it in depth.

In this study, network pharmacology strategy was adopted to study the mechanism of GGQLD against RVE. The active ingredients of GGQLD and their targets intersected with disease genes were identified to investigate network relationships between drugs, targets, and diseases through network pharmacology (Figure 1).

\section{Materials and Methods}

2.1. Reagent and Materials. Caco-2 cells were from Wuhan university cell bank (Wuhan, China). RV-WA was from the immunology institute of the Third Military Medical University (Chongqing, China). Hoechst dye was purchased from Solarbio Company (Beijing, China). Fura-3 fluorescence was purchased from Beyotime Company (Shanghai, China). The Pueraria lobate, Scutellaria baicalensis, Coptis chinensis, and Glycyrrhiza uralensis were purchased from Dongguan Sinopharm Group (product batch number: 20190501, Dongguan, China). High glucose medium and fetal bovine serum were purchased from Gibco Company (USA).

\subsection{Database Construction}

2.2.1. Compound Database Construction. We obtained information of GGQLD active ingredients from the traditional Chinese medicine systems pharmacology database and analysis platform (TCMSP) (http://sm.nwsuaf.edu.cn/lsp/ tcmsp.php), which is a comprehensive TCM database for network pharmacology and also provides ideal information of the ADME (absorption, distribution, metabolism, and excretion) properties of natural compounds [7]. With TCMSP, the active ingredients of GGQLD were mainly filtered through the criteria of oral bioavailability (OB) and drug-likeness (DL), which are important indicators to evaluate ADME properties through bioinformatics. The increase in $\mathrm{OB}$ can achieve maximum efficacy and minimal side effects [8]. DL has been widely used to screen compounds with undesirable properties [9]. The QSAR models based on multiple linear regression (MLR), partial least squares regression (PLS), and nonlinear support-vector machine regression (SVR) have good potential to predict OB
[10]. For DL, it is used in the drug design after evaluating whether it is similar to the physicochemical property and structural characteristics of existing drugs, which refers to molecular weight, one-dimensional descriptors, two-dimensional profiles, three-dimensional variables, and total positive and negative charges. In general, we calculate the DL by the Tanimoto coefficient, a formula based on database [11]: $f(x, y)=x y /|x|^{2}+|y|^{2}-x y$ (X represents the new numerator, and $y$ represents the overall parameters of all ingredients in Drug-Bank database). In this study, active ingredients with OB index $\geq 30 \%$ and DL index $\geq 0.18$ were selected as follow-up studies.

\subsubsection{Active Ingredients Targeted Protein and Genes Data-} base Construction. To obtain more detailed multicomponent drug targets, the next essential step was to find the specific targets where the drug ingredients play a pharmacodynamic role [12]. With the development of systems biology and computational methods, the ways to predict molecular targets are more abundant and reliable [13]. Here, we used a technique called similarity ensemble approach (http://sea.bkslab.org/) to obtain protein targeted by active ingredients from GGQLD, which is based on the chemical similarity among their ligands to match protein. Although the ligands are similar in the structure, both of them do not have an identical ligand [14]. Before searching the target protein limited to Homo sapiens in similarity ensemble approach, we had to get the canonical SMILES of the active ingredients as search term through PubChem platform (https://pubchem.ncbi.nlm.nih.gov/). Finally, we further used UniProtKB database (http://www.uniprot.org/) to verify the standard protein name and obtain the corresponding gene name as targets, which is the authority for the functional information of proteins [15].

\subsubsection{RVE Disease Target Genes Database Construction.} To maximize the total effective disease targets, we search them with the keyword "enteritis" in the database called GeneCards (https://www.genecards.org). From this database, we can obtain the relevant action enteritis-related genes of Homo sapiens, which included the all targets associated with RVE in particular.

\subsection{TCM-Intersecting Targets-Disease Network Construction.} The intersecting targets, displayed by Venn diagrams, were obtained between the targets of GGQLD ingredients and the RVE disease genes, which were used for the construction of PPI network and TCM-intersecting targets-disease network. First, based on the intersecting targets, PPI network was constructed to predict the interacting proteins through the STRING database (https://string-db.org/) with the multiple proteins function and the threshold of 0.95 interaction score. And the statistical interaction connectivity of proteins was used to determine the top 8 proteins. Next, in order to visualize the TCM-intersecting targets-disease network, we used Cytoscape software 3.7.1 to express the relationship between them in the form of a network diagram, where the 


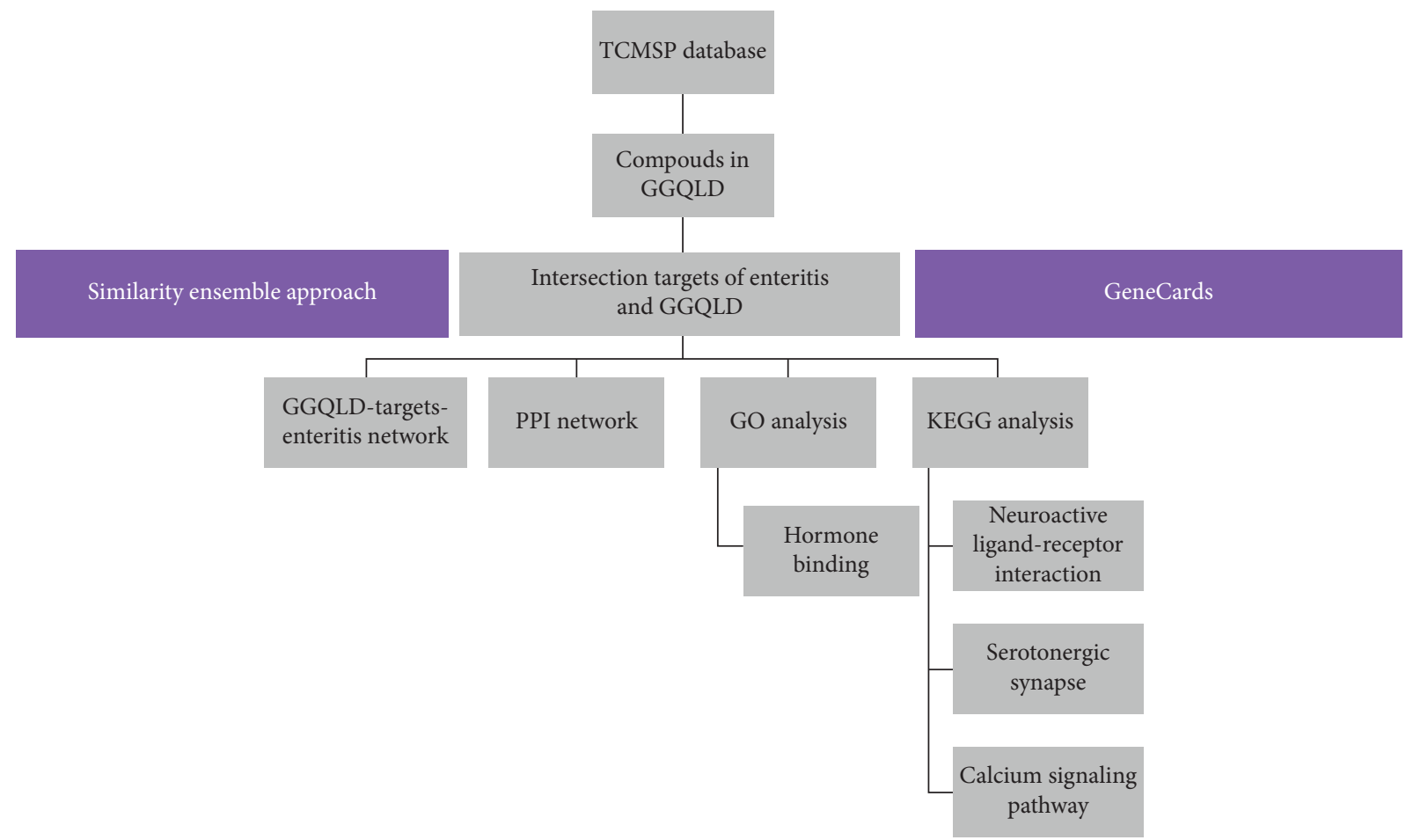

FIGURE 1: Flowchart of the systems pharmacology approach for hypothetical mechanism of GGQLD in treating RVE by ingredients collection, targets identification, and network analysis.

nodes represented diseases, ingredients, or targets, and the lines represented nodes' associations.

2.4. Biological Function Analysis. Through $\mathrm{R}$ language software (3.4.4) and Biocon-ductor's biocLite.R, the above intersecting targets were performed to GO enrichment analysis (from the biological processes) and KEGG enrichment analysis. GO (http://geneontology.org/) is used to annotate the genes of different species from three independent ontologies including biological process, molecular function, and cellular component [16]. Furthermore, KEGG (http://www.genome.jp/kegg/) is a database that has access to specifically analyze the distribution of intersecting targets in the pathway [17]. Finally, $P<0.05$ was selected as the standard of significant enrichment to obtain the biological processes and signaling pathways in which GGQLD participated in the anti-RVE pharmacological role. The Cytoscape software 3.7.1 was used to visualize and analyze their related targets and active ingredients interaction network.

\subsection{Functional Validation by Detection of Calcium Ions}

2.5.1. Preparation of GGQLD and Cytotoxicity Test. $15 \mathrm{~g}$ of Pueraria lobate, $9 \mathrm{~g}$ of Scutellaria baicalensis, $9 \mathrm{~g}$ of Coptis chinensis, and $6 \mathrm{~g}$ of Glycyrrhiza uralensis with 8 times weight of water were taken. Pueraria lobate was decocted for 20 min firstly, and the remaining herbs were decocted for a total of $30 \mathrm{~min}$. Besides, the half-inhibitory concentration (IC50) of GGQLD to Caco-2 cells was measured by MTT colorimetry.
2.5.2. Cell Culture and GGQLD Treatment. Caco-2 cells were routinely cultured in high-sugar DMEM medium containing $10 \%$ fetal bovine serum (containing 1\% antipenicillin and streptomycin). It was placed in an incubator at $37^{\circ} \mathrm{C}$ and $5 \%$ carbon dioxide. After it grew to a monolayer, it was routinely digested. Then, it was inoculated on the laser confocal special dish according to the density of $1 \times 10^{5}$ cells, which were divided into the control group, RV group, and GGQLD group. After $24 \mathrm{~h}$ of culture, the culture medium of the RV group and GGQLD group was removed. Then, $0.1 \mathrm{ml}$ $\mathrm{RV}$-WA with a virus titer of $10^{-4} / 0.1 \mathrm{ml}$ (firstly incubated with $10 \mu \mathrm{g} / \mathrm{ml}$ trypsin for $30 \mathrm{~min}$ at $37^{\circ} \mathrm{C}$ ) and $0.9 \mathrm{ml}$ serumfree DMEM culture medium were added, while the control group was added $1 \mathrm{ml}$ serum-free DMEM culture medium. After $2 \mathrm{~h}$ of infection, the culture medium of the control group and RV group was removed and $2 \mathrm{ml}$ serum-free DMEM culture medium was added for $24 \mathrm{~h}$. But the GGQLD group was added $2 \mathrm{ml}$ serum-free DMEM culture medium with $4.8 \mathrm{mg} / \mathrm{ml}$ of GGQLD.

2.5.3. Determination of Intracellular Calcium Ion Intensity. Upon completion, changes in intracellular calcium ion intensity were measured using a laser confocal microscope (Leica SP8) and fura-3 fluorescence loading technology. The cells were incubated in serum-free DMEM culture solution containing $5 \mu \mathrm{M}$ fluo-3/AM fluorescent probe at $37^{\circ} \mathrm{C}$ for $30 \mathrm{~min}$. The incubation solution was removed and then added $1 \mathrm{ml} 5 \mu \mathrm{g} / \mathrm{ml}$ Hoechst dye to be incubated for $5 \mathrm{~min}$. Finally, the cells were washed twice with PBS buffer solution. Finally, the fluorescence intensity of calcium ions was 
TABLE 1: The active ingredients of GGQLD in TCMSP database (their targets intersected with enteritis genes).

\begin{tabular}{|c|c|c|c|c|}
\hline Herbs & Compound name & $\mathrm{OB}$ & $\mathrm{DL}$ & PubChem ID \\
\hline \multirow{3}{*}{ Pueraria lobate } & Formononetin & 69.67 & 0.21 & 5280378 \\
\hline & $3^{\prime}$-Methoxydaidzein & 48.57 & 0.24 & 5319422 \\
\hline & Daidzein-4,7-diglucoside & 47.27 & 0.67 & 171292 \\
\hline \multirow{36}{*}{ Scutellaria baicalensis } & Acacetin & 34.97 & 0.24 & 5280442 \\
\hline & Wogonin & 30.68 & 0.23 & 5281703 \\
\hline & (2R)-7-Hydroxy-5-methoxy-2-phenyl-2,3-dihydrochromen-4-one & 55.23 & 0.2 & 821279 \\
\hline & Baicalein & 33.52 & 0.21 & 5281605 \\
\hline & $5,8,2^{\prime}$-Trihydroxy-7-methoxyflavone & 37.01 & 0.27 & 156992 \\
\hline & $5,7,3^{\prime}, 6^{\prime}$-Tetrahydroxy- $6,8,2^{\prime}$-trimethoxyflavone & 33.82 & 0.45 & 44258628 \\
\hline & Carthamidin & 41.15 & 0.24 & 188308 \\
\hline & $2,6,2^{\prime}, 4^{\prime}$-Tetrahydroxy-6'-methoxychalcone & 69.04 & 0.22 & 78385588 \\
\hline & Dihydrobaicalin_qt & 40.04 & 0.21 & 14135323 \\
\hline & Eriodyctiol & 41.35 & 0.24 & 373261 \\
\hline & Salvigenin & 49.07 & 0.33 & 161271 \\
\hline & 5,2',6'-Trihydroxy-7,8-dimethoxyflavone & 45.05 & 0.33 & 5322059 \\
\hline & $5,7,2^{\prime}, 6^{\prime}$-Tetrahydroxyflavone & 37.01 & 0.24 & 5321865 \\
\hline & Dihydrooroxylin A & 38.72 & 0.23 & 177032 \\
\hline & Skullcapflavone II & 69.51 & 0.44 & 124211 \\
\hline & Oroxylin A & 41.37 & 0.23 & 5320315 \\
\hline & Panicolin & 76.26 & 0.29 & 5320399 \\
\hline & 5,7,4'-Trihydroxy-8-methoxyflavone & 36.56 & 0.27 & 5322078 \\
\hline & NEOBAICALEIN & 104.34 & 0.44 & 124211 \\
\hline & $2 \beta$-Phenyl-2,3-dihydro-5,7-dihydroxy-6-methoxy-4H-1-benzopyran-4-one & 66.06 & 0.23 & 25721350 \\
\hline & $\beta$-Sitosterol & 36.91 & 0.75 & 222284 \\
\hline & 3-Epi- $\beta$-sitosterol & 36.91 & 0.75 & 12303645 \\
\hline & Norwogonin & 39.4 & 0.21 & 5281674 \\
\hline & 5,2'-Dihydroxy-6,7,8-trimethoxyflavone & 31.71 & 0.35 & 159029 \\
\hline & Ent-Epicatechin & 48.96 & 0.24 & 182232 \\
\hline & Stigmasterol & 43.83 & 0.76 & 5280794 \\
\hline & Coptisine & 30.67 & 0.86 & 72322 \\
\hline & $\operatorname{Bis}[(2 S)$-2-ethylhexyl]benzene-1,2-dicarboxylate & 43.59 & 0.35 & 7057920 \\
\hline & Supraene & 33.55 & 0.42 & 638072 \\
\hline & Diop & 43.59 & 0.39 & 33934 \\
\hline & Epiberberine & 43.09 & 0.78 & 160876 \\
\hline & Moslosooflavone & 44.09 & 0.25 & 188316 \\
\hline & 11,13-Eicosadienoic acid, methyl ester & 39.28 & 0.23 & 5365674 \\
\hline & 5,7,4'-Trihydroxy-6-methoxyflavanone & 36.63 & 0.27 & 5322074 \\
\hline & 5,7,4'-Trihydroxy-8-methoxyflavanone & 74.24 & 0.26 & 42608119 \\
\hline & Rivularin & 37.94 & 0.37 & 13889022 \\
\hline \multirow{12}{*}{ Coptis chinensis } & Berberine & 36.86 & 0.78 & 2353 \\
\hline & Obacunone & 43.29 & 0.77 & 119041 \\
\hline & Berberrubine & 35.74 & 0.73 & 72703 \\
\hline & (R)-Canadine & 55.37 & 0.77 & 443422 \\
\hline & Berlambine & 36.68 & 0.82 & 11066 \\
\hline & AC1O7GAS & 104.95 & 0.78 & 6604198 \\
\hline & Magnograndiolide & 63.71 & 0.19 & 5319198 \\
\hline & Palmidin A & 35.36 & 0.65 & 5320384 \\
\hline & Palmatine & 64.6 & 0.65 & 19009 \\
\hline & Quercetin & 46.43 & 0.28 & 5280343 \\
\hline & Worenine & 45.83 & 0.87 & 20055073 \\
\hline & Moupinamide & 86.71 & 0.26 & 5280537 \\
\hline
\end{tabular}


TABle 1: Continued.

\begin{tabular}{|c|c|c|c|c|}
\hline Herbs & Compound name & OB & $\mathrm{DL}$ & PubChem ID \\
\hline & Inermine & 75.18 & 0.54 & 91510 \\
\hline & DFV & 32.76 & 0.18 & 114829 \\
\hline & Mairin & 55.38 & 0.78 & 64971 \\
\hline & Glycyrol & 90.78 & 0.67 & 5320083 \\
\hline & Jaranol & 50.83 & 0.29 & 5318869 \\
\hline & Medicarpin & 49.22 & 0.34 & 336327 \\
\hline & Isorhamnetin & 49.6 & 0.31 & 5281654 \\
\hline & 3-Epi- $\beta$-sitosterol & 36.91 & 0.75 & 12303645 \\
\hline & Lupiwighteone & 51.64 & 0.37 & 5317480 \\
\hline & 7-Methoxy-2-methyl isoflavone & 42.56 & 0.2 & 911486 \\
\hline & Formononetin & 69.67 & 0.21 & 5280378 \\
\hline & Calycosin & 47.75 & 0.24 & 5280448 \\
\hline & Kaempferol & 41.88 & 0.24 & 5280863 \\
\hline & Naringenin & 59.29 & 0.21 & 932 \\
\hline & Shinflavanone & 31.79 & 0.72 & 197678 \\
\hline & Glyasperin B & 65.22 & 0.44 & 480784 \\
\hline & Glyasperin F & 75.84 & 0.54 & 392442 \\
\hline & Glyasperin C & 45.56 & 0.4 & 480859 \\
\hline & Isotrifoliol & 31.94 & 0.42 & 5318679 \\
\hline & (E)-1-(2,4-Dihydroxyphenyl)-3-(2,2-dimethylchromen-6-yl)prop-2-en-1-one & 39.62 & 0.35 & 10881804 \\
\hline & Kanzonol W & 50.48 & 0.52 & 15380912 \\
\hline & (2S)-6-(2,4-Dihydroxyphenyl)-2-(2-hydroxypropan-2-yl)-4-methoxy-2,3-dihydrofuro[3,2-g]chromen-7-one & 60.25 & 0.63 & 637112 \\
\hline & Semilicoisoflavone B & 48.78 & 0.55 & 5481948 \\
\hline & Glepidotin A & 44.72 & 0.35 & 5281619 \\
\hline & Glepidotin B & 64.46 & 0.34 & 442411 \\
\hline & Phaseolinisoflavan & 32.01 & 0.45 & 4484952 \\
\hline & Glypallichalcone & 61.6 & 0.19 & 5317768 \\
\hline & 8-(6-Hydroxy-2-benzofuranyl)-2,2-dimethyl-5-chromenol & 58.44 & 0.38 & 10542808 \\
\hline & Licochalcone B & 76.76 & 0.19 & 5318999 \\
\hline & Licochalcone G & 49.25 & 0.32 & 49856081 \\
\hline & Licoarylcoumarin & 59.62 & 0.43 & 10090416 \\
\hline & Licoricone & 63.58 & 0.47 & 5319013 \\
\hline & Gancaonin A & 51.08 & 0.4 & 5317478 \\
\hline & Gancaonin B & 48.79 & 0.45 & 5317479 \\
\hline & Licorice glycoside E & 32.89 & 0.27 & 42607811 \\
\hline & Gancaonin L & 66.37 & 0.41 & 14604077 \\
\hline & Gancaonin M & 30.49 & 0.41 & 14604078 \\
\hline & Gancaonin $\mathrm{O}$ & 44.15 & 0.41 & 14604081 \\
\hline & Glycyrin & 52.61 & 0.47 & 480787 \\
\hline & Licocoumarone & 33.21 & 0.36 & 503731 \\
\hline & Licoisoflavone & 41.61 & 0.42 & 5281789 \\
\hline & Licoisoflavone B & 38.93 & 0.55 & 5481234 \\
\hline & Licoisoflavanone & 52.47 & 0.54 & 392443 \\
\hline & Shinpterocarpin & 80.3 & 0.73 & 10336244 \\
\hline \multirow{46}{*}{ Glycyrrhiza uralensis } & (E)-3-[3,4-Dihydroxy-5-(3-methylbut-2-enyl)phenyl]-1-(2,4-dihydroxyphenyl)prop-2-en-1-one & 46.27 & 0.31 & 11267805 \\
\hline & Liquiritin & 65.69 & 0.74 & 503737 \\
\hline & Licopyranocoumarin & 80.36 & 0.65 & 122851 \\
\hline & 3,22-Dihydroxy-11-oxo-delta(12)-oleanene-27-alpha-methoxycarbonyl-29-oic acid & 34.32 & 0.55 & 195396 \\
\hline & Glyzaglabrin & 61.07 & 0.35 & 5317777 \\
\hline & Glabridin & 53.25 & 0.47 & 124052 \\
\hline & Glabranin & 52.9 & 0.31 & 124049 \\
\hline & Glabrene & 46.27 & 0.44 & 480774 \\
\hline & Glabrone & 52.51 & 0.5 & 5317652 \\
\hline & Hedysarimcoumestan B & 48.14 & 0.43 & 11558452 \\
\hline & 3,5-Dihydroxy-13,14-dimethoxy-8,17-dioxatetracyclo[8.7.0.0(2),.0(1) (1),(1)]hepta-deca-1(10),2,4,6,11,13,15-hepta-en-9-one & 62.9 & 0.53 & 1160239 \\
\hline & Eurycarpin A & 43.28 & 0.37 & 5317300 \\
\hline & Glycyroside & 37.25 & 0.79 & 44257223 \\
\hline & (-)-Medicocarpin & 40.99 & 0.95 & 23724664 \\
\hline & Sigmoidin-B & 34.88 & 0.41 & 73205 \\
\hline & (2R)-7-Hydroxy-2-(4-hydroxyphenyl)-2,3-dihydrochromen-4-one & 71.12 & 0.18 & 928837 \\
\hline & Isobavachin & 36.57 & 0.32 & 193679 \\
\hline & Isoglycyrol & 44.7 & 0.84 & 124050 \\
\hline & Isolicoflavonol & 45.17 & 0.42 & 5318585 \\
\hline & Isoformononetin & 38.37 & 0.21 & 3764 \\
\hline & 1-Methoxyphaseollidin & 69.98 & 0.64 & 480873 \\
\hline & Quercetin der & 46.45 & 0.33 & 52761906 \\
\hline & $3^{\prime}$-Hydroxy-4'-O-methylglabridin & 43.71 & 0.57 & 15228662 \\
\hline & Licochalcone A & 40.79 & 0.29 & 5318998 \\
\hline & $3^{\prime}$-Methoxyglabridin & 46.16 & 0.57 & 15228663 \\
\hline & $4^{\prime}$-Methoxyglabridin & 36.21 & 0.52 & 9927807 \\
\hline & Icos-5-enoic acid & 30.7 & 0.2 & 3349565 \\
\hline & Kanzonol F & 32.47 & 0.89 & 101666840 \\
\hline & 6-Prenylated eriodictyol & 39.22 & 0.41 & 13845972 \\
\hline & $7,2^{\prime}, 4^{\prime}$-Trihydroxy-5-methoxy-3-arylcoumarin & 83.71 & 0.27 & 25015742 \\
\hline & 7-Acetoxy-2-methylisoflavone & 38.92 & 0.26 & 268208 \\
\hline & (2S)-3', $4^{\prime}, 5,7$-Tetrahydroxy-8-prenylflavanone & 53.79 & 0.4 & 13845973 \\
\hline & Gadelaidic acid & 30.7 & 0.2 & 5460988 \\
\hline & Vestitol & 74.66 & 0.21 & 92503 \\
\hline & Gancaonin G & 60.44 & 0.39 & 480780 \\
\hline & Gancaonin $\mathrm{H}$ & 50.1 & 0.78 & 5481949 \\
\hline & Licoagrocarpin & 58.81 & 0.58 & 15840593 \\
\hline & Glyasperin M & 72.67 & 0.59 & 101664572 \\
\hline & Glycyrrhiza flavonol A & 41.28 & 0.6 & 5317765 \\
\hline & Licoagroisoflavone & 57.28 & 0.49 & 636883 \\
\hline & 18- $\alpha$-Hydroxyglycyrrhetic acid & 41.16 & 0.71 & 101280181 \\
\hline & Odoratin & 49.95 & 0.3 & 13965473 \\
\hline & Phaseol & 78.77 & 0.58 & 44257530 \\
\hline & Xambioona & 54.85 & 0.87 & 14769500 \\
\hline & Dehydroglyasperin $\mathrm{c}$ & 53.82 & 0.37 & 480775 \\
\hline & Quercetin & 46.43 & 0.28 & 5280343 \\
\hline
\end{tabular}


TABle 2: Names and PubChem IDs of GGQLD active ingredients (number of intersecting targets $>5$ ).

\begin{tabular}{|c|c|c|c|c|}
\hline Herbs & Compound name & PubChem ID & OB & DL \\
\hline \multirow{3}{*}{ Pueraria lobate } & $3^{\prime}$-Methoxydaidzein & 5319422 & 48.57 & 0.24 \\
\hline & Daidzein-4,7-diglucoside & 171292 & 47.27 & 0.67 \\
\hline & Acacetin & 5280442 & 34.97 & 0.24 \\
\hline \multirow{11}{*}{ Scutellaria baicalensis } & Wogonin & 5281703 & 30.68 & 0.23 \\
\hline & (2R)-7-Hydroxy-5-methoxy-2-phenyl-2,3-dihydrochromen-4-one & 821279 & 55.23 & 0.2 \\
\hline & Baicalein & 5281605 & 33.52 & 0.21 \\
\hline & 5,8,2'-Trihydroxy-7-methoxyflavone & 156992 & 37.01 & 0.27 \\
\hline & $2,6,2^{\prime}, 4^{\prime}$-Tetrahydroxy- $6^{\prime}$-methoxychalcone & 78385588 & 69.04 & 0.22 \\
\hline & $\beta$-Sitosterol & 222284 & 36.91 & 0.75 \\
\hline & Bis $[(2 S)$-2-ethylhexyl]benzene-1,2-dicarboxylate & 7057920 & 43.59 & 0.35 \\
\hline & Supraene & 638072 & 33.55 & 0.42 \\
\hline & Diop & 33934 & 43.59 & 0.39 \\
\hline & 11,13-Eicosadienoic acid methyl ester & 5365674 & 39.28 & 0.23 \\
\hline & (R)-Canadine & 443422 & 55.37 & 0.77 \\
\hline \multirow{4}{*}{ Coptis chinensis } & Quercetin & 5280343 & 46.43 & 0.28 \\
\hline & Moupinamide & 5280537 & 86.71 & 0.26 \\
\hline & Mairin & 911486 & 55.38 & 0.78 \\
\hline & Formononetin & 5280378 & 69.67 & 0.21 \\
\hline \multirow{4}{*}{ Glycyrrhiza uralensis } & Glypallichalcone & 5317768 & 61.6 & 0.19 \\
\hline & Icos-5-enoic acid & 3349565 & 30.7 & 0.2 \\
\hline & 7-Acetoxy-2-methylisoflavone & 268208 & 38.92 & 0.26 \\
\hline & Gadelaidic acid & 5460988 & 30.7 & 0.2 \\
\hline
\end{tabular}

measured with the laser confocal microscope at the excitation wavelength of $488 \mathrm{~nm}$ and the absorption wavelength of $515-565 \mathrm{~nm}$.

\section{Results}

3.1. Composition Analysis. After searching, 142 active ingredients that passed the $\mathrm{OB}$ and DL filters were identified from the TCMSP database. However, only 130 of these ingredients showed the shared targets across disease genes (shown in Table 1), and the active ingredients with the number of intersecting targets $>5$ are listed in Table 2 .

\subsection{TCM-Intersecting Targets-Disease Network Analysis.} Finally, we found 7149 enteritis-related targets and 552 GGQLD active ingredient targets, with 366 intersections (Figure 2(a)). The 366 intersecting targets were imported into the String database to obtain the PPI network targeting anti-RVE (Figure 2(b)). In this network, as shown in Figure 2(c), the top 8 genes with the highest node connectivity were EP300, AKT1, HSP90AA1, CTNNB1, EGFR, CDK1, HRAS, and KAT2B. Among them, EP300 has 31 connections with other genes, and 7 other genes have more than 15 connectivities. Finally, with the intersection genes as a hub, we constructed a network diagram of GGQLDintersecting targets-active ingredients (represented by PubChem ID) RVE, which could clearly determine the relationship between them (Figure 2(d)).

3.3. Biological Functional Analysis. In terms of biological processes, the intersecting targets demonstrate multiple functions (Figure 3(a)). The redder the color in the figure, the smaller the $P$ value, and the stronger the representativeness. The most interesting one is hormone binding with ID 0042562. The function is interpreted as selectively and noncovalently interacting with any of the hormones to influence the metabolism or behavior of other cells with hormone-functional receptors.

In the signal pathway, its importance evaluation criteria were similar to the GO diagram (Figure 3(b)). The results showed the following pathways with reference value: neuroactive ligand-receptor interaction with ID hsa04080, serotonergic synapse with ID hsa04726, calcium signaling pathway with ID hsa04020, and so on. These pathways were concentrated in the related expression of neural active substances, especially in the serotonergic system.

3.4. Active Ingredients-Interaction Targets-Pathways Network Analysis. Through Cytoscape software 3.7.1, the active ingredients and interaction targets related to the biological process horizon binding and the neuroactive ligand receptor interaction, serotonergic synapse, and calcium signaling pathway were visualized as the network (Figure 4). Among them, the active ingredients ( $\mathrm{R})$-canadine, moupinamide, and formononetin, respectively, targeted 17,17 , and 11 targets. It is speculated that these active ingredients may be the key ingredients for GGQLD to exert anti-RVE effects through the above functional pathways. In addition, the serotonin receptor subfamily (HTR1A, HTR2B, and HTR7), the adrenergic receptor subfamily (ADRA2A, ADRA2B, ADRB1, ADRB2, and ADRB3), the cholinergic receptor subfamily (CHRM1, CHRM2, and CHRM3), and the dopamine receptor subfamily (DRD1, DRD2, DRD3, DRD4, and DRD5), respectively, corresponded to $8,7,7$, and 7 


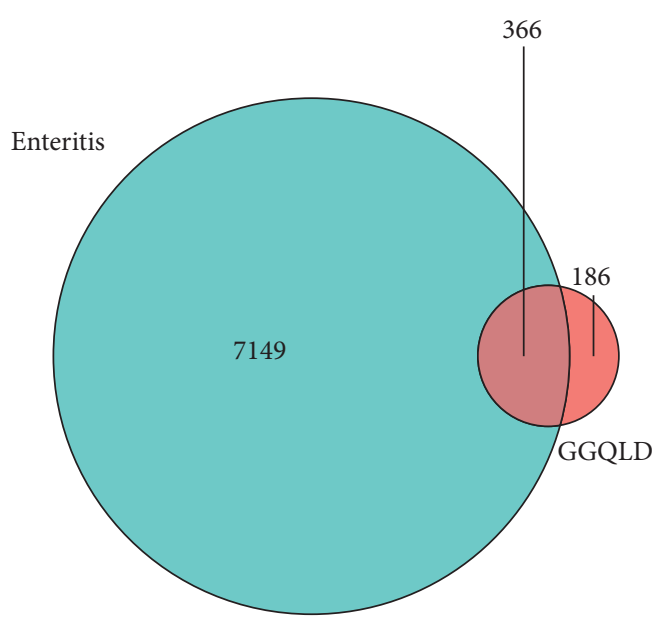

(a)

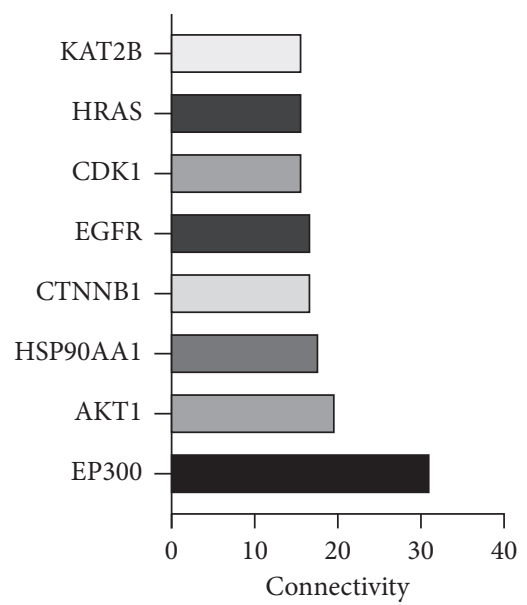

(c)

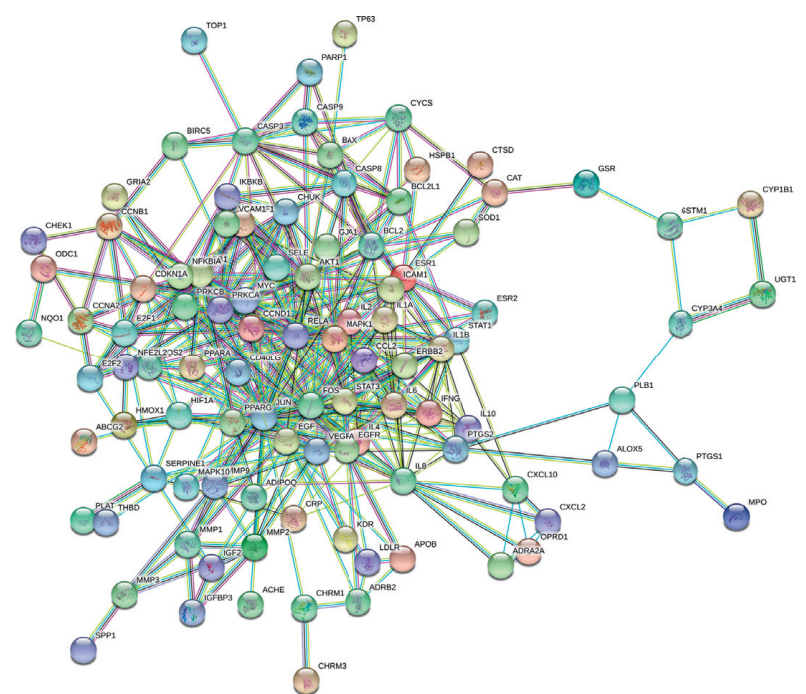

(b)

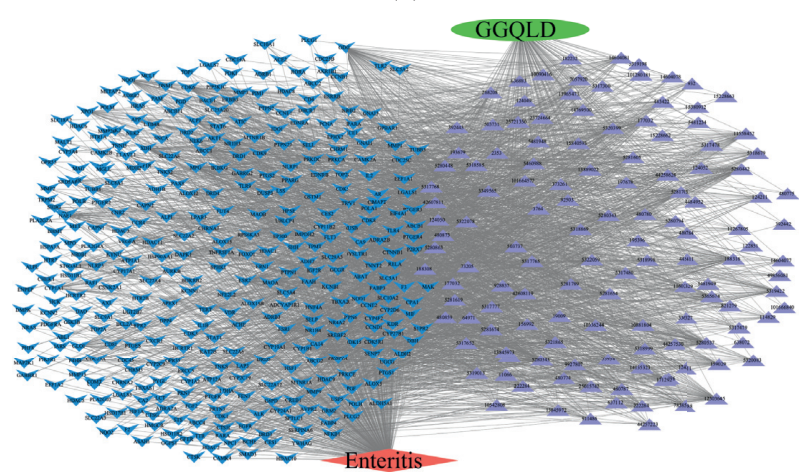

(d)

Figure 2: TCM-intersecting targets-disease network diagram: (a) intersecting targets of enteritis and GGQLD. There are 7149 enteritis-related targets (blue section) and 552 active ingredients targets (red section), with 366 intersections; (b) PPI network of intersecting targets; (c) genes with top 8 PPI network connectivities. The abscissa represents the number of connections with other genes, and the ordinate represents the name of the gene; (d) network of GGQLD ( $\square$ ), enteritis $(\square)$, intersecting targets ( $\square$ ), and active ingredients $(\square)$ in molecular level. In the diagram, intersecting targets were represented by gene IDs and active ingredients were represented by the PubChem IDs.

active ingredients. It is speculated that these targets may be the key parts for GGQLD to exert anti-RVE efficacy through the above functional pathways.

3.5. GGQLD Inhibited the Release of Calcium Ions in Caco-2 Cells after $R V$ Infection. After applying $4.8 \mathrm{mg} / \mathrm{ml}$ of GGQLD (IC50: $16.21 \mathrm{mg} / \mathrm{ml}$, as shown in Figure 5(a)) to RV infected Caco- 2 cells 24 hours, we observe the result with laser confocal microscope. As shown in Figure 5(b), intracellular calcium ion fluorescence intensity in Caco- 2 cells infected with the RV was significantly stronger than the control group. However, the GGQLD group could reduce the fluorescence intensity of intracellular calcium ions in RV-infected Caco-2 cells, which showed that GGQLD had access to decrease the release of intracellular calcium ions.

\section{Discussion}

4.1. Analysis of Active Ingredients of GGQLD. From the TCMSP database, 130 active ingredients of GGQLD were found to be related to RVE, which mainly included the types of flavonoids, alkaloids, phenyl ester, and fatty acids. The flavonoids have the abilities to destroy the integrity of RV structure and protein synthesis, which has the potentials of anti-RV drug research [18, 19]. An ingredient called 


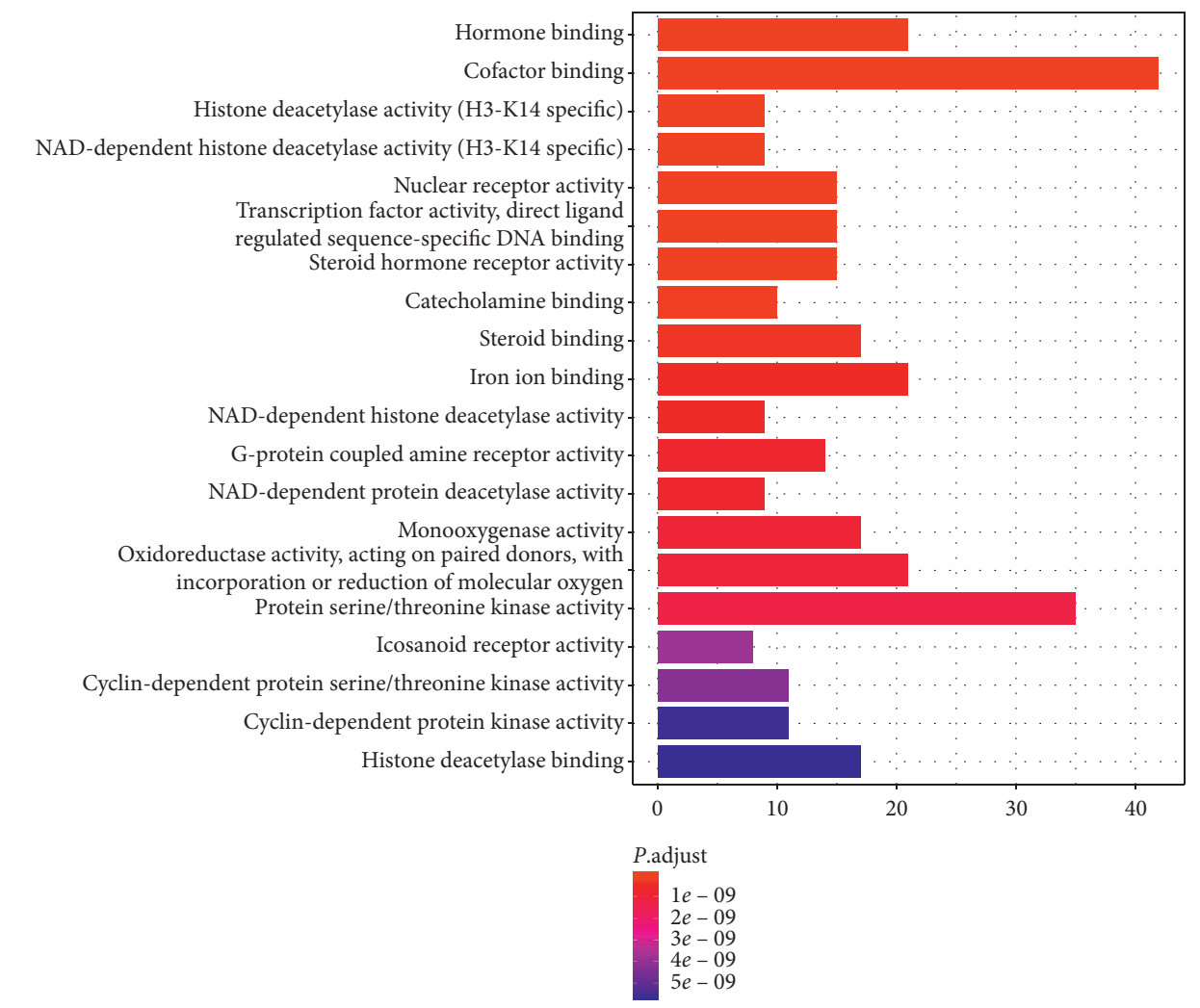

(a)

Neuroactive-ligand-receptor interaction

Prostate cancer

Serotonergic synapse Calcium signaling pathway

Proteoglycans in cancer

Thyroid hormone signaling pathway EGFR tyrosine kinase inhibitor resistance

cAMP signaling pathway Bile secretion

Glioma

VEGF signaling pathway

AGE-RAGE signaling pathway in diabetic complications

Arachidonic acid metabolism

Cell cycle

Acute myeloid leukemia

Viral carcinogenesis

Cocaine addiction

Steroid hormone biosynthesis

HIF-1 signaling pathway

PI3K-Akt signaling pathway

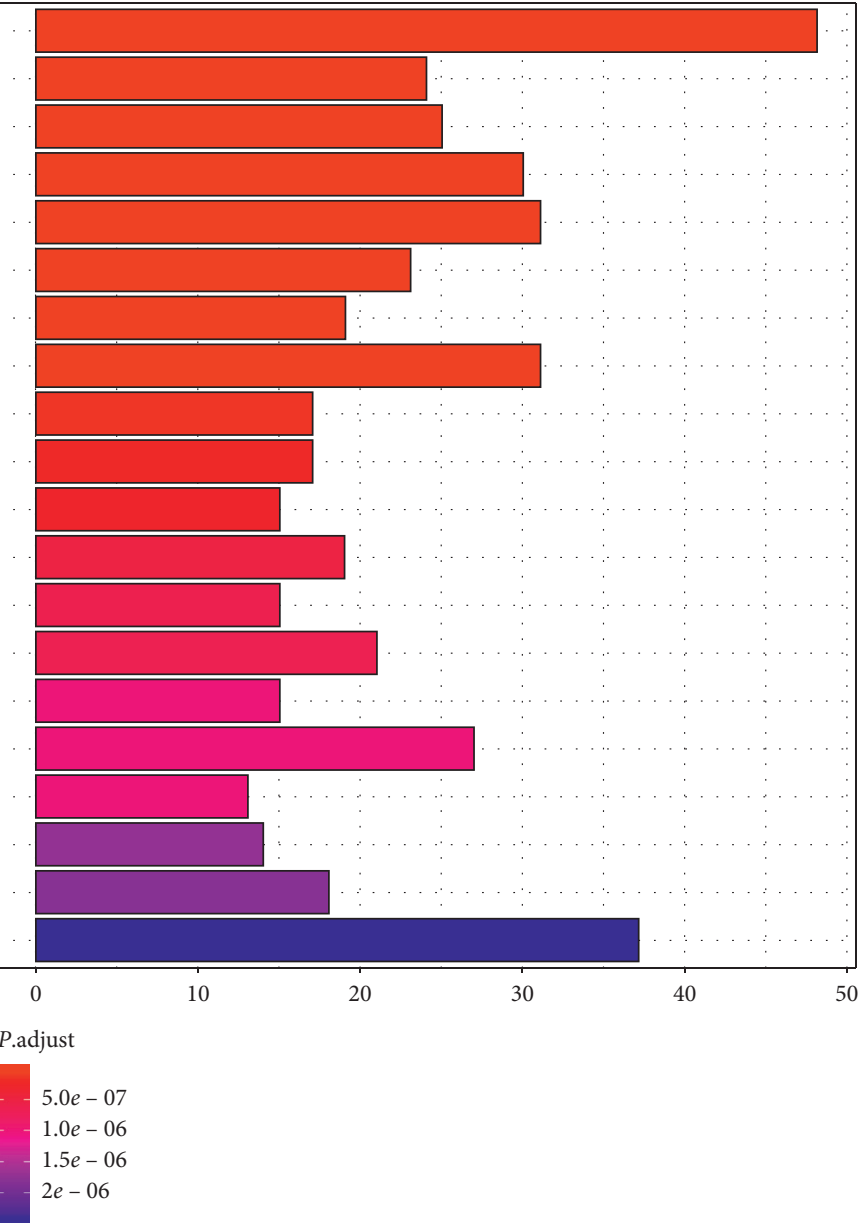

(b)

FIGURE 3: Biological function analysis of GGQLD anti-RVE targets: (a) GO enrichment analysis of the intersecting targets' biological process; (b) KEGG enrichment analysis of intersecting targets' signaling pathway. 


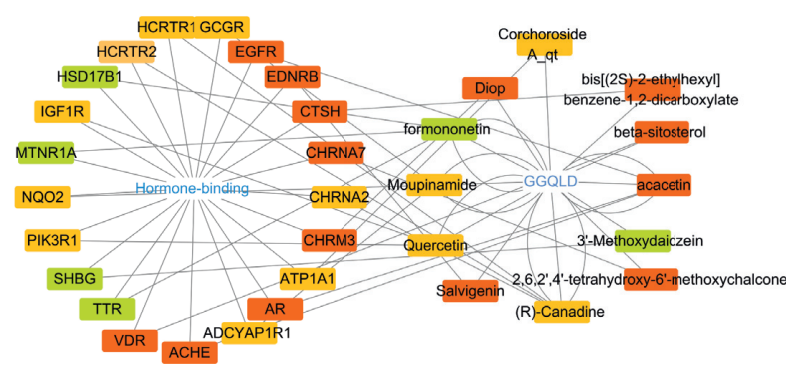

(a)

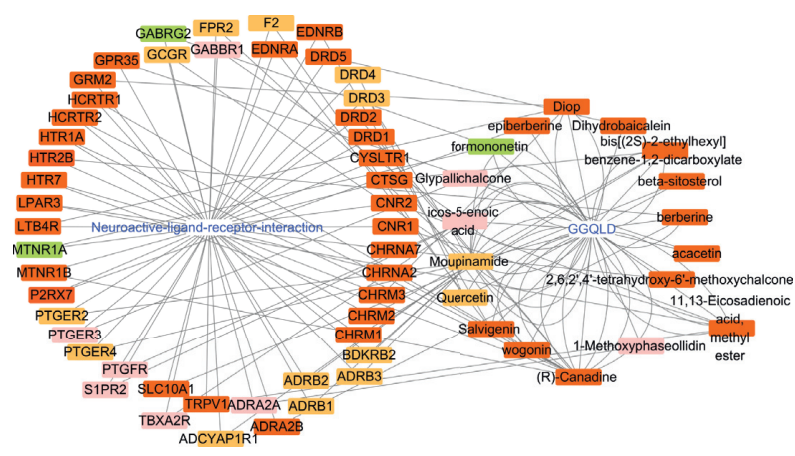

(c)

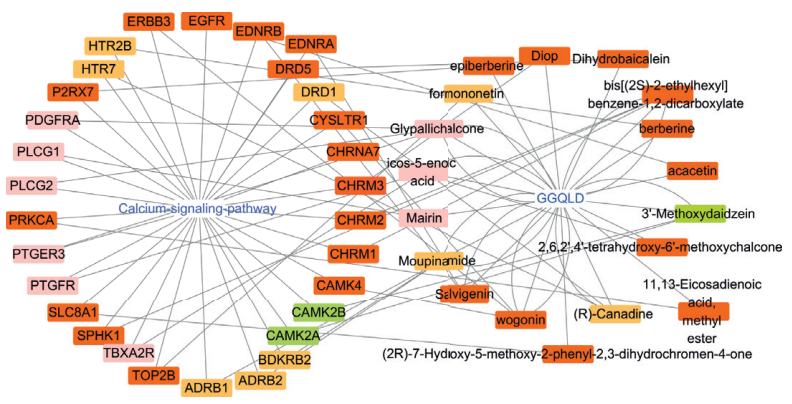

(b)

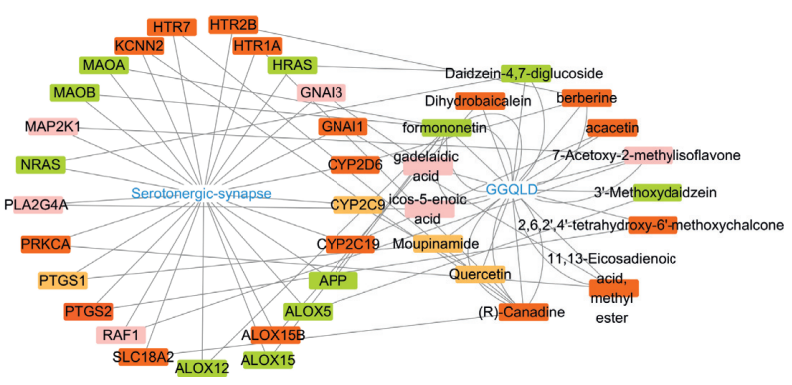

(d)

FIGURE 4: Network of interaction targets-active ingredients-pathways from the perspective of key function and pathways: (a) hormone binding; (b) calcium signaling pathway; (c) neuroactive ligand-receptor interaction; (d) serotonergic synapse (Pueraria lobate, Scutellaria baicalensis, Coptis chinensis, and Glycyrrhiza uralensis are showed by $\square, \square, \square$, and $\square)$.

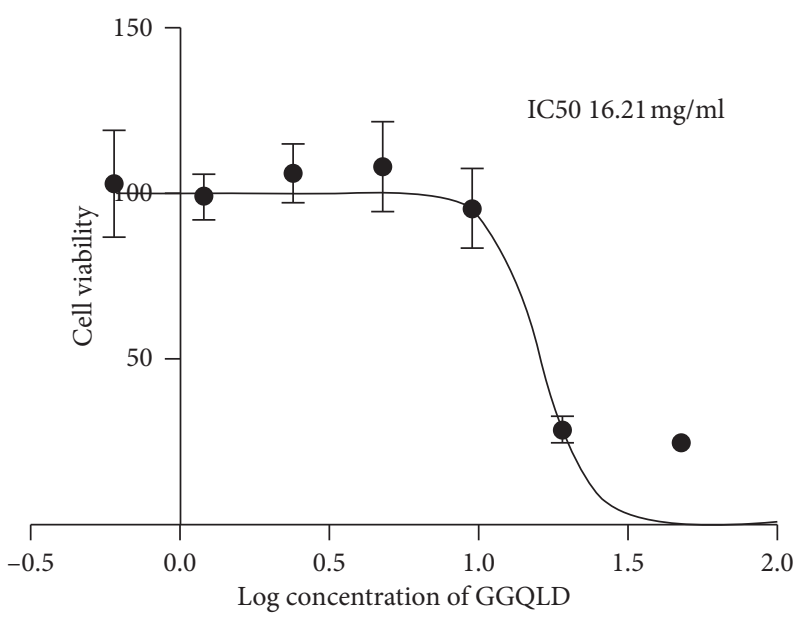

(a)

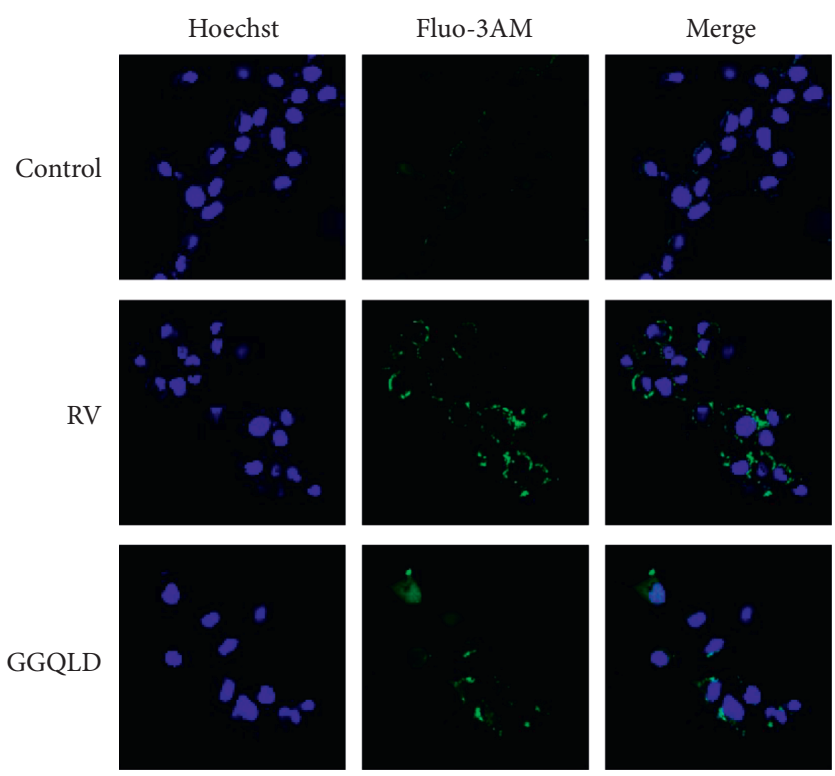

(b)

FIGURE 5: Intracellular calcium ion intensity of Caco-2 cells: (a) the IC50 of GGQLD to Caco-2 cells; (b) the calcium ion intensity of the control group, RV group, and GGQLD group. Blue fluorescence represents the nucleus, and the green fluorescence represents the calcium ions. 


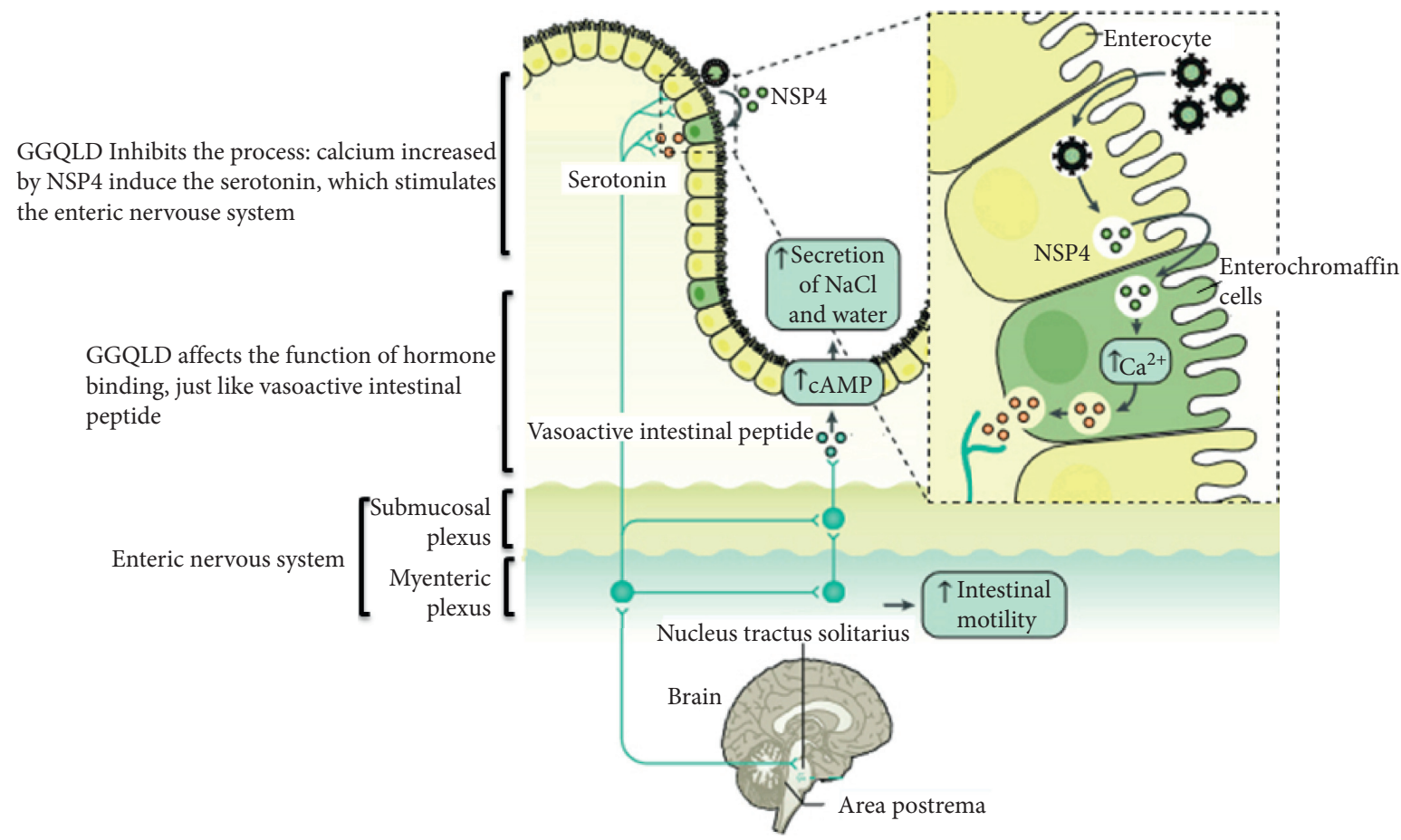

FIGURE 6: The hypothetical mechanism of GGQLD in treating RVE. First, GGQLD reduced the release of calcium ions from intestinal cells caused by RV, thereby inhibiting the secretion of serotonin. It could impede the impulse of the nervous system of the intestine to reduce the promotion effect of vasoactive intestinal peptide on cAMP, which finally balanced the intestinal water and salt metabolism and eased RVE diarrhea.

licocoumarone, for example, has the ability to suppress viral RNA synthesis in TF-104 cells [20]. From Figure 4, we also found that flavonoids such as formononetin, acacetin, wogonin, and quercetin were distributed in key pathways and target multiple disease genes. In addition, other active ingredients named (R)-canadine, icos-5-enoic-acid, moupinamide, and bis[(2S)-2-ethylhexyl]-benzene-1,2-dicarboxylate also had potential research value. Overall, we could find that Scutellaria baicalensis was the most influential Chinese medicine, containing a variety of key active ingredients.

4.2. Closely Related Target Analysis from the PPI Network. The PPI network showed that the deeper potential targets of GGQLD were related to proliferation and transcription. As the histone acetyltransferase, EP300 and KAT2B play a causative role in regulating transcription through internal lysine acetylation of multiple proteins and a rapid and reversible regulatory mechanism [21]. In addition, HSP90AA1, respectively, promotes autophagy and inhibits apoptosis through the PI3K/Akt/mTOR pathway and JNK/ P38 pathway [22]. AKT1 (a downstream target of phosphatidylinositol 3-kinase) and CTNNB1 (a central part in Wnt/beta-catenin pathway) both have access to regulate a number of cellular proliferation and differentiation [23, 24]. And with the active autophosphorylation of receptor tyrosine kinase, EGFR could initiate a cascade of downstream signaling pathways involved in regulating cellular differentiation [25]. As for CDK1, it is required for the transition from the G2 phase into mitosis, which affects cell proliferation a lot [26]. In short, these targets with high connectivity are closely related to cell proliferation and differentiation.

4.3. The Impact of Hormone Binding. Gastrointestinal hormones are the basic regulators of intestinal absorption, metabolism, and homeostasis and play important roles in intestinal fitness [27]. Similar to GGQLD for damp-heat syndrome of TCM, ZHIXIE decoction may improve the efficacy of damp-heat RV infection enteritis by regulating gastrointestinal hormones [28]. In a variety of gastrointestinal hormones, vasoactive intestinal peptide in gastrointestinal hormones is associated with the pathways described below. In general, vasoactive intestinal peptide is synthesized under the activation of serotonin receptors on intrinsic primary afferent nerves that compose the myenteric plexus [29], which can cause diarrhea through up-regulated cAMP [30]. Interestingly, as shown in Figure 6, recent studies have reported that serotonin homeostasis can be destroyed by RV infection [31, 32].

\subsection{The Analysis of Neuroactive Ligand-Receptor Interaction,} Serotonergic Synapse, and Calcium Signaling Pathway. From the perspective of disease, RVE seems to be related to the intestinal nervous system [33]. For example, the enteric neurons have a lot to do with gastrointestinal functions and age, which are also related to RV [34]. And as described in the hormone binding analysis, recent studies have 
demonstrated that RVE has potential mechanisms that enteric nervous system was affected by serotonin, which was corresponding to the results of neuroactive ligand-receptor interaction and serotonergic synapse obtained by analysis [32]. Furthermore, it also shown that increased intracellular calcium level mediated by NSP4 could induce the serotonin of enterochromaffin cells, which can stimulate the intestinal nervous system and ultimately enhance the irritable bowel movement $[31,35,36]$. Besides, we found that RV infection in infants and young children would disrupt calcium homeostasis to increased calcium ion concentration in intestinal epithelial cells, which was closely related to infection [37]. It was corresponding to the results of calcium ion signaling pathway, which indicated that GGQLD may act on the calcium ion channel to achieve therapeutic effect. In terms of experimental results, this study also preliminarily found that GGQLD could inhibit the condition that RV increased calcium release in Caco-2 cells. In short, we supposed GGQLD mainly achieves antidiarrhea effects on the calcium ion release, serotonin homeostasis, gastrointestinal hormones, and intestinal nerve system (Figure 6).

\section{Conclusions}

GGQLD had 130 active ingredients in the treatment of RVE, such as (R)-canadine, formononetin, and moupinamide. They targeted 366 genes to regulate the secretion of gastrointestinal hormones of intestinal epithelial cells and stabilize the secretion of calcium ions and serotonin, among which serotonin receptors, adrenergic receptors, cholinergic receptors, and dopamine receptors were potential key points. In summary, this study reveals the anti-RVE mechanism of GGQLD through multicomponent-multitarget-multipathway and provided new ideas for comprehensive and in-depth clarification of the mechanism of GGQLD in the treatment of RVE.

\section{Data Availability}

The data used to support the findings of this study are available from the corresponding author upon request.

\section{Disclosure}

Peicheng Zhong and Lijun Song are the co-first authors.

\section{Conflicts of Interest}

The authors declare that there are no conflicts of interest regarding the publication of this paper.

\section{Authors' Contributions}

Peicheng Zhong, Lijun Song, and Mengyue Gao were responsible for the research concept, design, and literature retrieval. Peicheng Zhong, Zuli Zhao, and Xiaotong Wang were responsible for data analysis and interpretation. Wenpan Tan and Huanqian Lu drafted the paper. Qian Lan and Wenchang Zhao have made extensive modifications. All authors participated in the analysis and interpretation of the data and passed the final paper. Peicheng Zhong and Lijun Song made equal contributions to this work.

\section{Acknowledgments}

This study was funded by the National Natural Science Foundation of China (nos. 81973548 and 81473401) and Key Project of Social Science and Technology Development of Dongguan (no. 20185071521658).

\section{References}

[1] C. Li, Q. Li, R. Liu et al., "Medicinal herbs in the prevention and treatment of osteoporosis," The American Journal of Chinese Medicine, vol. 42, no. 1, pp. 1-22, 2014.

[2] F. Tang, Q. Tang, Y. Tian, Q. Fan, Y. Huang, and X. Tan, "Network pharmacology-based prediction of the active ingredients and potential targets of Mahuang Fuzi Xixin decoction for application to allergic rhinitis," Journal of Ethnopharmacology, vol. 176, pp. 402-412, 2015.

[3] A. L. Hopkins, "Network pharmacology: the next paradigm in drug discovery," Nature Chemical Biology, vol. 4, no. 11, pp. 682-690, 2008.

[4] L. Song, W. Zhao, X. Tan, M. Yang, and J. Luo, "Effect of Gegen Qinlian decoction on rotavirus-inducing diarrhea in neonatal mice," Chinese Journal of Information on Traditional Chinese Medicine, vol. 20, no. 7, pp. 35-37, 2013.

[5] J. Shen, J. Chen, Y. Chen, and L. Zhao, "Clinical observation on 20 cases of rotavirus diarrhea treated with Gegen Qinlian decoction," Chinese Medicine Modern Distance Education of China, vol. 11, no. 2, pp. 91-93, 2013.

[6] S. E. Crawford, S. Ramani, J. E. Tate et al., "Rotavirus infection," Nature Reviews Disease Primers, vol. 3, p. 17083, 2017.

[7] J. Ru, P. Li, J. Wang et al., "TCMSP: a database of systems pharmacology for drug discovery from herbal medicines," Journal of Cheminformatics, vol. 6, no. 1, p. 13, 2014.

[8] R. Wang, D. P. Conner, and B. V. Li, "Bioavailability and bioequivalence aspects of oral modified-release drug products," The AAPS Journal, vol. 19, no. 2, pp. 360-366, 2017.

[9] S. Tian, J. Wang, Y. Li, D. Li, L. Xu, and T. Hou, “The application of in silico drug-likeness predictions in pharmaceutical research," Advanced Drug Delivery Reviews, vol. 86, pp. 2-10, 2015.

[10] X. Xu, W. Zhang, C. Huang et al., "A novel chemometric method for the prediction of human oral bioavailability," International Journal of Molecular Sciences, vol. 13, no. 6, pp. 6964-6982, 2012.

[11] L. Liu, B. Du, H. Zhang et al., "A network pharmacology approach to explore the mechanisms of Erxian decoction in polycystic ovary syndrome," Chinese Medicine, vol. 13, no. 1, p. 46, 2018.

[12] M. Schenone, V. Dančík, B. K. Wagner, and P. A. Clemons, "Target identification and mechanism of action in chemical biology and drug discovery," Nature Chemical Biology, vol. 9, no. 4, pp. 232-240, 2013.

[13] J. S. Fang, A. L. Liu, and G. H. Du, "Research advance in the drug target prediction based on chemoinformatics," Yao Xue Xue Bao, vol. 49, no. 10, pp. 1357-1364, 2014.

[14] M. J. Keiser, B. L. Roth, B. N. Armbruster, P. Ernsberger, J. J. Irwin, and B. K. Shoichet, "Relating protein pharmacology by ligand chemistry," Nature Biotechnology, vol. 25, no. 2, pp. 197-206, 2007.

[15] C. T. UniProt, "UniProt: the universal protein knowledgebase," Nucleic Acids Research, vol. 46, no. 5, p. 2699, 2018. 
[16] M. Ashburner, C. A. Ball, J. A. Blake et al., "Gene Ontology: tool for the unification of biology," Nature Genetics, vol. 25, no. 1, pp. 25-29, 2000.

[17] M. Tanabe and M. Kanehisa, "Using the KEGG database resource," Current Protocols in Bioinformatics, vol. 11, no. 1, 2012.

[18] G. R. Gandhi, P. G. Barreto, B. d. S. Lima et al., "Medicinal plants and natural molecules with in vitro and in vivo activity against rotavirus: a systematic review," Phytomedicine, vol. 23, no. 14 , pp. 1830-1842, 2016.

[19] H. Huang, D. Liao, L. Liang, L. Song, and W. Zhao, "Genistein inhibits rotavirus replication and upregulates AQP4 expression in rotavirus-infected Caco-2 cells," Archives of Virology, vol. 160, no. 6, pp. 1421-1433, 2015.

[20] H.-J. Kwon, H.-H. Kim, Y. B. Ryu et al., "In vitro anti-rotavirus activity of polyphenol compounds isolated from the roots of Glycyrrhiza uralensis," Bioorganic \& Medicinal Chemistry, vol. 18, no. 21, pp. 7668-7674, 2010.

[21] M.-H. Kuo and C. D. Allis, "Roles of histone acetyltransferases and deacetylases in gene regulation," Bioessays, vol. 20, no. 8, pp. 615-626, 1998.

[22] X. Xiao, W. Wang, Y. Li et al., "HSP90AA1-mediated autophagy promotes drug resistance in osteosarcoma," Journal of Experimental \& Clinical Cancer Research, vol. 37, no. 1, p. 201, 2018.

[23] S. Boyault, D. S. Rickman, A. de Reyniès et al., "Transcriptome classification of HCC is related to gene alterations and to new therapeutic targets," Hepatology, vol. 45, no. 1, pp. 42-52, 2007.

[24] R. T. Moon, "Wnt/beta-catenin pathway," Science Signaling, vol. 2005, no. 271, 2005.

[25] D. Singh, B. Kumar Attri, R. Kaur Gill, and J. Bariwal, "Review on EGFR inhibitors: critical updates," Mini-Reviews in Medicinal Chemistry, vol. 16, no. 14, pp. 1134-1166, 2016.

[26] M. Malumbres, "Cyclin-dependent kinases," Genome Biology, vol. 15, no. 6, p. 122, 2014.

[27] M. Wabitsch, "Gastrointestinal hormones induced the birth of endocrinology," Developmental Biology of Gastrointestinal Hormones, vol. 32, pp. 1-7, 2017.

[28] M. Li, Y. Wang, N. Luo, and J. Cui, "Effect of Chinese herbal Zhixie Decoction on immune function and Gastrointestinal hormones in patients with damp-heat rotavirus infection," Northwest Pharmaceutical Journa, vol. 34, no. 2, pp. 241-244, 2019.

[29] M. Hagbom, C. Istrate, D. Engblom et al., "Rotavirus stimulates release of serotonin (5-HT) from human enterochromaffin cells and activates brain structures involved in nausea and vomiting," PLoS Pathogens, vol. 7, no. 7, 2011.

[30] C. D. Rao, P. P. Maiya, and M. A. Babu, "Non-diarrhoeal increased frequency of bowel movements (IFoBM-ND): enterovirus association with the symptoms in children," $B M J$ Open Gastroenterology, vol. 1, no. 1, 2014.

[31] S. Bialowas, M. Hagbom, J. Nordgren et al., "Rotavirus and serotonin cross-talk in diarrhoea," PLoS One, vol. 11, no. 7, 2016.

[32] S. Kordasti, H. Sjovall, O. Lundgren, and L. Svensson, "Serotonin and vasoactive intestinal peptide antagonists attenuate rotavirus diarrhoea," Gut, vol. 53, no. 7, pp. 952-957, 2004.

[33] A. P. Morris and M. K. Estes, "VIII. Pathological consequences of rotavirus infection and its enterotoxin," American Journal of Physiology-Gastrointestinal and Liver Physiology, vol. 281, no. 2, pp. G303-G310, 2001.
[34] M. J. Saffrey, "Cellular changes in the enteric nervous system during ageing," Developmental Biology, vol. 382, no. 1, pp. 344-355, 2013.

[35] A. L. Chang-Graham, J. L. Perry, A. C. Strtak et al., "Rotavirus calcium dysregulation manifests as dynamic calcium signaling in the cytoplasm and endoplasmic reticulum," Scientific Reports, vol. 9, no. 1, p. 10822, 2019.

[36] T. Pham, J. L. Perry, T. L. Dosey, A. H. Delcour, and J. M. Hyser, "The rotavirus NSP4 viroporin domain is a calcium-conducting ion channel," Scientific Reports, vol. 7, Article ID 43487, 2017.

[37] J. M. Hyser, M. R. Collinson-Pautz, B. Utama, and M. K. Estes, "Rotavirus disrupts calcium homeostasis by NSP4 viroporin activity," mBio, vol. 1, no. 5, 2010. 